\title{
Special Classes of Coprime Irregular Graphs
}

\author{
P. Sankara Narayanan, Assistant Professor, Kalasalingam Academy of Research and Education \\ Krishnankoil \& India, sankarmath4@gmail.com \\ S. Saravanakumar, Assistant Professor, Kalasalingam Academy of Research and Education \\ Krishnankoil \& India, saramath25@gmail.com
}

Abstract: An $k$-edge-weighting of a graph $G=(V, E)$ is a mapping $\varphi: E(G) \rightarrow\{1,2,3, \ldots k\}$, where $k$ is a positive integer. The sum of the edge-weighting appearing on the edges incident at the vertex $v$ under the edge-weighting $\varphi$ and is denoted by $S_{\varphi}(\mathrm{v})$. An $k$ edge-weighting of $G$ is a coprime irregular edge-weighting if $\operatorname{gcd}\left(S_{\varphi}(\mathrm{u}), S_{\varphi}(\mathrm{v})\right)=1$ for every pair of adjacent vertices $u$ and $v$ in G. A graph $G$ admits a coprime irregular edge-weighting is called a coprime irregular graph. In this paper, we discuss the coprime irregular edge-weighting for some special classes of graphs.

Keywords -Irregular edge weighting, friendship graph, Dutch windmill graph.

\section{INTRODUCTION}

By a graph $G=(V, E)$, we mean a finite, undirected graph with neither loops nor multiple edges. For graph theoretic terminology we refer to Chartrand and Lesniak [3].

A graph labeling is an assignment of integers to the vertices or edges or both with respect to some conditions. A detailed survey of graph labeling is given by Gallian [4]. Basically the class of edge-weighting problems can be divided into two parts. The first one is the proper edge-weighting of a graph $\mathrm{G}$, that is, a weighting of $\mathrm{G}$ in which no two incident edges receive the same labels and the other one is the nonproper edge-weighting in which the incident edges may receive same labels. For detailed study of this kind of edgeweightings, one may refer [1], [2], [5]. With respect to the non-proper edge-weighting, I. Sahul Hamid et.al[6] introduced the concept of equitable irregular edgeweighting of graphs and in which the authors discussed some properties of equitable irregular graphs. Motivated by the definition of equitable irregular graphs, S. Saravanakumar introduced the concept of coprime irregular graphs in [7] and found some coprime irregular graphs. Continuation of this work, in this paper, we prove that some special classes of graphs such as friendship graphs, Dutch windmill graphs, double quadrilateral snakes and pan graphs are coprime irregular.

\section{COPRIME IRREGUlAR GRAPHS}

This section aims to prove that some special classes of graphs such as Friendship graphs, Dutch windmill graphs, Double quadrilateral graphs and Pan graphs are coprime irregular.

Definition 2.1. The friendship graph $F_{n}$ can be constructed by joining $\mathrm{n}$ copies of the cycle graph $C_{3}$ with a common vertex.
Theorem 2.2. The friendship graph $F_{n}(\mathrm{n} \geq 3)$ is coprime irregular graph for all $\mathrm{n}$.

Proof. Let V $\left(F_{n}\right)=\left\{\mathrm{v}_{0}, \mathrm{v}_{1}, \mathrm{v}_{2}, \ldots, \mathrm{v}_{\mathrm{n}}, \mathrm{u}_{1}, \mathrm{u}_{2}, \ldots, \mathrm{u}_{\mathrm{n}}\right\}$ and $\mathrm{E}\left(\mathrm{F}_{\mathrm{n}}\right)=\left\{v_{0} v_{i}, v_{0} u_{i}, v_{i} u_{i}: 1 \leq \mathrm{i} \leq \mathrm{n}\right\}$. We prove this theorem in the following cases.

Case (i): $n$ is odd

For all $\mathrm{i}=1,2,3, \ldots, \mathrm{n}$, let $\varphi\left(\mathrm{v}_{0} \mathrm{v}_{\mathrm{i}}\right)=\frac{n-1}{2}, \varphi\left(\mathrm{v}_{0} \mathrm{u}_{\mathrm{i}}\right)=\frac{n+1}{2}$ and $\varphi\left(\mathrm{v}_{\mathrm{i}} \mathrm{u}_{\mathrm{i}}\right)=\frac{n+3}{2}$. Then $S_{\varphi}\left(\mathrm{v}_{0}\right)=n^{2}, S_{\varphi}\left(\mathrm{v}_{\mathrm{i}}\right)=\mathrm{n}+1$ and $S_{\varphi}\left(\mathrm{u}_{\mathrm{i}}\right)=\mathrm{n}+2$ for all $\mathrm{n}$. Clearly, the weights of any two adjacent vertices of $F_{n}$ is relatively prime and so $F_{n}$ is coprime irregular (For instance, the graph $\mathrm{F}_{3}$ and its coprime irregular edge weighting $\varphi$ is given in Figure 1.). This proves Case (i).

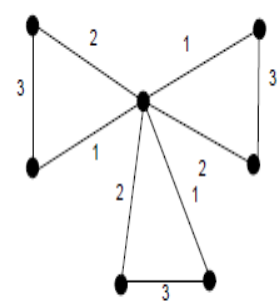

Figure 1: The graph $F_{3}$ and its coprime irregular edge-weighting under $\phi$

Case (ii): $n$ is even

For all $\mathrm{i}=1,2,3, \ldots, \mathrm{n}$, let $\varphi\left(\mathrm{v}_{0} \mathrm{v}_{\mathrm{i}}\right)=\frac{n-2}{2}, \varphi\left(\mathrm{v}_{0} \mathrm{u}_{\mathrm{i}}\right)=\frac{n+2}{2}$ and $\varphi\left(\mathrm{v}_{\mathrm{i}} \mathrm{u}_{\mathrm{i}}\right)=\frac{n}{2}$. Then $S_{\varphi}\left(\mathrm{v}_{0}\right)=n^{2}, S_{\varphi}\left(\mathrm{v}_{\mathrm{i}}\right)=\mathrm{n}-1$ and $S_{\varphi}$ $\left(u_{i}\right)=n+1$ for all $n$. It is easy to verify that the weight of any two adjacent vertices of $F_{n}$ are relatively prime (The graph $F_{4}$ and its coprime irregular edge weighting $\varphi$ is illustrated in Figure 2.) 


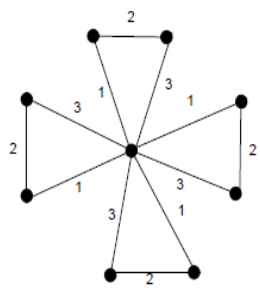

Figure 2: The graph $F_{4}$ and its coprime irregular edge-weighting under $\phi$

Definition 2.3. A double quadrilateral snake $\mathrm{DQ}_{\mathrm{n}}$ is a graph consists of two quadrilateral snakes that have a common path.

Theorem 2.4. For all $n \geq 3$, the graph double quadrilateral snake $\mathrm{DQ}_{\mathrm{n}}$ is coprime irregular.

Proof. Let $V\left(D Q_{n}\right)=\left\{v_{1}, v_{2}, \ldots, v_{n+1}\right\} \cup\left\{u_{i}, w_{i}, x_{i}, y_{i}: 1 \leq i\right.$ $\leq n\}$ and $E\left(D Q_{n}\right)=\left\{v_{i} u_{i}, u_{i} w_{i}, v_{i+1} w_{i}, v_{i} x_{i}, x_{i} y_{i}, v_{i+1} y_{i}: 1 \leq i\right.$ $\leq n\}$. Define an edge weighting $\varphi$ of $D Q_{n}$ as follows.

$$
\varphi\left(v_{i} v_{i+1}\right)=\left\{\begin{array}{lr}
2, & \text { if } \mathrm{i} \equiv 1(\bmod 3) \\
1, & \text { if } \mathrm{i} \equiv 2(\bmod 3) \\
4, & \text { other wise }
\end{array}\right.
$$

and $\varphi\left(v_{i} u_{i}\right)=\varphi\left(v_{i} x_{i}\right)=1$ and $\varphi\left(u_{i} w_{\mathrm{i}}\right)=\varphi\left(x_{i} y_{i}\right)=2$ and $\varphi\left(\mathrm{v}_{\mathrm{i}+1} \mathrm{w}_{\mathrm{i}}\right)=3$ for all $\mathrm{i}=1,2, \ldots, \mathrm{n}-1$. Further, assign $\varphi\left(\mathrm{v}_{\mathrm{i}+1} \mathrm{y}_{\mathrm{i}}\right)=3$ for all $\mathrm{i}=1,2,3 \ldots, \mathrm{n}-2$ and for $\mathrm{i}=\mathrm{n}-1$, assign

$$
\varphi\left(v_{i+1} y_{i}\right)= \begin{cases}3, & \text { if } i \equiv 2(\bmod 3) \\ 5, & \text { if } i \equiv 0(\bmod 3) \\ 6, & \text { if } i \equiv 1(\bmod 3)\end{cases}
$$

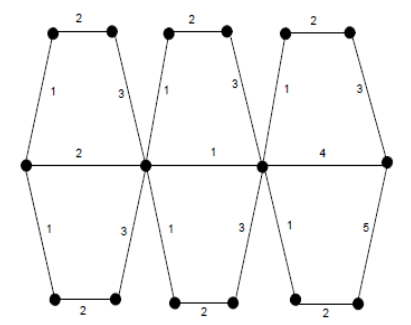

Figure 3: The graph $D Q_{4}$ and its coprime irregular edge-weighting under $\phi$ Clearly, $S_{\varphi}\left(\mathrm{v}_{1}\right)=4$ and then for all $\mathrm{i}=2,3 \ldots, \mathrm{n}-1$, we have

$$
S_{\varphi}\left(\mathrm{v}_{\mathrm{i}}\right)= \begin{cases}11, & \text { if } \mathrm{i} \equiv 2(\bmod 3) \\ 13, & \text { if } \mathrm{i} \equiv 0(\bmod 3) \\ 14, & \text { if } \mathrm{i} \equiv 1(\bmod 3)\end{cases}
$$

and

$$
S_{\varphi}\left(\mathrm{v}_{\mathrm{n}}\right)=\left\{\begin{array}{cc}
7, & \text { if } \mathrm{i} \equiv 0(\bmod 3) \\
12, & \text { if } \mathrm{i} \equiv 1(\bmod 3) \\
11, & \text { if } \mathrm{i} \equiv 2(\bmod 3)
\end{array}\right.
$$

Also, for all $\mathrm{i}=1,2,3 \ldots, \mathrm{n}-1$, we have $S_{\varphi}\left(\mathrm{u}_{\mathrm{i}}\right)=S_{\varphi}\left(\mathrm{x}_{\mathrm{i}}\right)=3$ and for all $\mathrm{i}=1,2,3 \ldots, \mathrm{n}-2$, we get $S_{\varphi}\left(\mathrm{y}_{\mathrm{i}}\right)=5$. Further, for $\mathrm{i}=\mathrm{n}-1$,

$$
S_{\varphi}\left(\mathrm{y}_{\mathrm{i}}\right)= \begin{cases}5, & \text { if } \mathrm{i} \equiv 2(\bmod 3) \\ 7, & \text { if } \mathrm{i} \equiv 0(\bmod 3) \\ 8, & \text { if } \mathrm{i} \equiv 1(\bmod 3)\end{cases}
$$

Clearly, the weights of any two adjacent vertices of $D Q_{n}$ are relatively prime. Therefore $D Q_{n}$ is coprime irregular graph [The graph $D Q_{4}$ and the coprime irregular edge weighting $\varphi$ is illustrated in Figure 3].

Definition 2.5. The Dutch windmill graph $D_{n}^{(m)}$ can be constructed by joining $\mathrm{m}$ copies of the cycle graph $\mathrm{C}_{4}$ with a common vertex

Theorem 2.6. The Dutch windmill graph $D_{n}^{(m)}(\mathrm{m} \geq 3)$ is coprime irregular for all $\mathrm{m}$.

Proof. Let $\mathrm{V}\left(D_{n}^{(m)}\right)=\left\{v_{0}, v_{i}, u_{i}, w_{i}: 1 \leq \mathrm{i} \leq \mathrm{m}\right\}$ and $\mathrm{E}\left(D_{n}^{(m)}\right)=\left\{\mathrm{v}_{0} \mathrm{v}_{\mathrm{i}}, \mathrm{v}_{\mathrm{i}} \mathrm{u}_{\mathrm{i}}, \mathrm{u}_{\mathrm{i}} \mathrm{w}_{\mathrm{i}}, \mathrm{v}_{0} \mathrm{w}_{\mathrm{i}} ; 1 \leq \mathrm{i} \leq \mathrm{m}\right\}$. Now, let us define an edge weighting $\varphi$ of $D_{n}^{(m)}$ as follows. We prove this theorem in the following two cases.

Case(i): $m$ is odd

For all $\mathrm{i}=1,2, \ldots, m$, define $\varphi\left(\mathrm{v}_{0} \mathrm{v}_{\mathrm{i}}\right)=\frac{m-1}{2}$, $\varphi\left(\mathrm{v}_{\mathrm{i}} \mathrm{u}_{\mathrm{i}}\right)=\frac{m+3}{2}$ and $\varphi\left(\mathrm{u}_{\mathrm{i}} \mathrm{W}_{\mathrm{i}}\right)=\varphi\left(\mathrm{v}_{0} \mathrm{~W}_{\mathrm{i}}\right)=\frac{m+1}{2}$.

Then $S_{\varphi}\left(\mathrm{v}_{0}\right)=m^{2}, S_{\varphi}\left(\mathrm{u}_{\mathrm{i}}\right)=\mathrm{m}+2, S_{\varphi}\left(\mathrm{v}_{\mathrm{i}}\right)=S_{\varphi}\left(\mathrm{w}_{\mathrm{i}}\right)=\mathrm{m}+1$. Thus any two adjacent vertices of $D_{n}^{(m)}$ whose weights are coprime and so $\varphi$ is a coprime irregular edge-weighting of $D_{n}^{(m)}$. Hence the Case(i) is follows.

[The graph $D_{4}^{(3)}$ and its coprime irregular edge weighting $\varphi$ is illustrated in Figure 4].

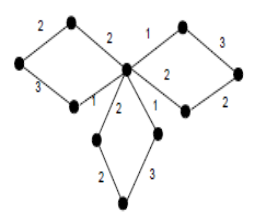

Figure 4: The graph $D_{4}^{(3)}$ and its coprime irregular edge-weighting under $\phi$ Case(ii): $m$ is even

For all $i=1,2, \ldots, m$, define $\varphi\left(\mathrm{v}_{0} \mathrm{v}_{\mathrm{i}}\right)=\frac{m-2}{2}$, $\varphi\left(v_{i} u_{i}\right)=\varphi\left(u_{i} w_{i}\right)=\frac{m}{2}$ and $\varphi\left(v_{0} w_{i}\right)=\frac{m+1}{2}$. Then $S_{\varphi}\left(v_{0}\right)=m^{2}$, $S_{\varphi}\left(u_{\mathrm{i}}\right)=\mathrm{m}, S_{\varphi}\left(v_{\mathrm{i}}\right)=\mathrm{m}-1, S_{\varphi}\left(w_{i}\right)=m+1$. Clearly, any two adjacent vertices of $D_{n}^{(m)}$ whose weights are relatively prime and thus $\varphi$ is coprime irregular edge weighting of $D_{n}^{(m)}$. This proves Case(ii). (The graph $D_{4}^{(4)}$ and its coprime irregular edge weighting $\varphi$ is illustrated in Figure 5).

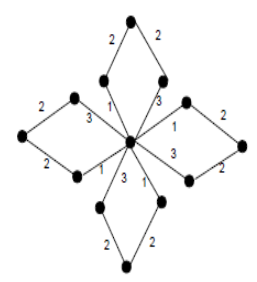

Figure 5: The graph $D_{4}^{(4)}$ and its coprime irregular edge-weighting under $\phi$

Definition 2.7. The pan graph $(P n)_{n}$ is the graph obtained by joining a cycle graph $\mathrm{C}_{\mathrm{n}}$ to a singleton graph $\mathrm{K}_{1}$ with a bridge.

Theorem 2.8. For all $n \geq 4$, the pan graph $(\mathrm{Pn})_{\mathrm{n}}$ is coprime irregular. 
Proof. We prove the result in the following two cases. Let $\mathrm{V}\left((\mathrm{Pn})_{\mathrm{n}}\right)=\left\{\mathrm{v}_{0}, \mathrm{v}_{1}, \mathrm{v}_{2}, \ldots, \mathrm{v}_{\mathrm{n}}\right\}$ and $\mathrm{E}\left((\mathrm{Pn})_{\mathrm{n}}\right)=\left\{\mathrm{v}_{\mathrm{i}} \mathrm{v}_{\mathrm{i}+1}: 1 \leq \mathrm{i}\right.$ $\leq \mathrm{n}-1\} \cup\left\{\mathrm{v}_{1} \mathrm{v}_{\mathrm{n}}\right\} \cup\left\{\mathrm{v}_{1} \mathrm{v}_{0}\right\}$. Consider the edge weighting $\varphi$ of $\left((\mathrm{Pn})_{\mathrm{n}}\right)$ define as follows.

Case(i): $n$ is even

For all $1 \leq \mathrm{i} \leq \mathrm{n}-1$, let

$$
\varphi\left(\mathrm{v}_{\mathrm{i}} \mathrm{v}_{\mathrm{i}+1}\right)=\left\{\begin{array}{l}
1, \text { if } \mathrm{i} \equiv 0(\bmod 4) \\
2, \text { if } \mathrm{i} \equiv 1(\bmod 4) \\
3, \text { if } \mathrm{i} \equiv 2(\bmod 4) \\
4, \text { if } \mathrm{i} \equiv 3(\bmod 4)
\end{array}\right.
$$

and

$$
\varphi\left(\mathrm{v}_{1} \mathrm{v}_{\mathrm{n}}\right)= \begin{cases}1, & \text { if } \mathrm{n} \equiv 0(\bmod 4) \\ 3, & \text { if } \mathrm{n} \equiv 2(\bmod 4)\end{cases}
$$

and assign $\varphi\left(\mathrm{v}_{1} \mathrm{v}_{0}\right)=1$. Then, we have

$$
S_{\varphi}\left(\mathrm{v}_{1}\right)= \begin{cases}4, & \text { if } \mathrm{n} \equiv 0(\bmod 4) \\ 6, & \text { if } \mathrm{n} \equiv 2(\bmod 4)\end{cases}
$$

and for all $\mathrm{i}=2,3, \ldots, \mathrm{n}$,

$$
S_{\varphi}\left(\mathrm{v}_{\mathrm{i}}\right)=\left\{\begin{array}{cc}
3, & \text { if } \mathrm{i} \equiv 1(\bmod 4) \\
5 & \text { if } \mathrm{i} \equiv 0(\operatorname{or}) 2(\bmod 4) \\
7, & \text { if } \mathrm{i} \equiv 3(\bmod 4)
\end{array}\right.
$$

and $S_{\varphi}\left(\mathrm{v}_{0}\right)=1$.

This proves that the weights of any two adjacent vertices of $(\mathrm{Pn})_{\mathrm{n}}$ is relatively prime and so the graph $(\mathrm{Pn})_{\mathrm{n}}$ is coprime irregular .[For instance, the graph $(\mathrm{Pn})_{4}$ and the coprime irregular egde weighting under $\varphi$ is given Figure 6. ]

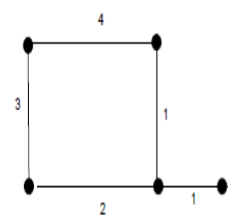

Figure 6: The graph $(P n)_{4}$ and its coprime irregular edge-weighting under $\phi$

Case(ii): $n$ is odd

For all $1 \leq \mathrm{i} \leq \mathrm{n}-1$, let

$$
\varphi\left(v_{i} v_{i+1}\right)=\left\{\begin{array}{l}
1, \text { if } \mathrm{i} \equiv 1(\bmod 4) \\
2, \text { if } \mathrm{i} \equiv 2(\bmod 4) \\
3, \text { if } \mathrm{i} \equiv 3(\bmod 4) \\
4, \text { if } \mathrm{i} \equiv 0(\bmod 4)
\end{array}\right.
$$

and

$$
\varphi\left(v_{1} v_{n}\right)= \begin{cases}1, & \text { if } \mathrm{n} \equiv 1(\bmod 4) \\ 3, & \text { if } \mathrm{n} \equiv 3(\bmod 4)\end{cases}
$$

and also assign

$$
\varphi\left(v_{1} v_{0}\right)=\left\{\begin{array}{ll}
3, & \text { if } \mathrm{n} \equiv 3(\bmod 4) \\
5, & \text { if } \mathrm{n} \equiv 1(\bmod 4)
\end{array} .\right.
$$

Then, we have

$$
S_{\varphi}\left(v_{0}\right)= \begin{cases}3, & \text { if } \mathrm{n} \equiv 1(\bmod 4) \\ 5, & \text { if } \mathrm{n} \equiv 3(\bmod 4)\end{cases}
$$

and for all $\mathrm{i}=2,3, \ldots, \mathrm{n}$,

$$
S_{\varphi}\left(\mathrm{v}_{\mathrm{i}}\right)=\left\{\begin{array}{cc}
3, & \text { if } \mathrm{i} \equiv 2(\bmod 4) \\
5 & \text { if } \mathrm{i} \equiv 1(\operatorname{or}) 3(\bmod 4) \\
7, & \text { if } \mathrm{i} \equiv 0(\bmod 4)
\end{array}\right.
$$

Now it is easy to observe that the weights of any two adjacent vertices of $(\mathrm{Pn})_{\mathrm{n}}$ is coprime and so $\varphi$ is coprime irregular edge weighting of $(\mathrm{Pn})_{\mathrm{n}}$. Hence the graph $(\mathrm{Pn})_{\mathrm{n}}$ is

coprime irregular. [For example, the graph $(\mathrm{Pn})_{3}$ and its coprime irregular edge weighting $\varphi$ is shown in Figure 7. ]

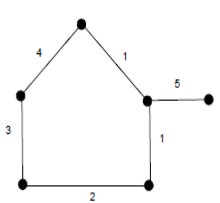

Figure 7: The graph $(P n)_{5}$ and its coprime irregular edge-weighting under $\phi$

\section{CONCLUSION}

In this paper, we proved some special classes of graphs such as friendship graphs, Dutch windmill graph, double quadrilateral snakes and Pan Graphs are coprime irregular graphs. However, there is a wide scope for further research in this topic. Here we list some of them.

1. Obtaining a characterization of coprime irregular graphs.

2. Characterizing trees which are coprime irregular is worthy trying.

3. Obtain more families of coprime irregular graphs using graph operations.

\section{ACKNOWLEDGMENT}

The authors wish to thank the management and head of the department of the mathematics for their indebted support for this research work.

\section{REFERENCES}

[1] D. Amar, "Irregularity strength of regular graphs of large degree. Combinatorics and algorithms (Jerusalem, 1988)", Discr. Math.,114 (1993), no 1 3, 917.

[2]G. Chartrand, M. S. Jacobson, J. Lehel, O. R. Oellermann, S. Ruiv and F. Saba, "Irregular networks, Congr. Numer.”, 64 (1988), 197210.

[3] G. Chartrand and Lesniak, "Graphs and Digraphs, Fourth Edition, CRC press, Boca Raton (2005).

[4] J.A. Gallian, "A dynamic survey of graph labeling", Electron. J . Combinator., 15(2008), 190.

[5] M. Karonski, T. Luczak and A. Thomson, "Edge weights and vertex colours". J.Combinator. Theory B, 91(2004), 151-157.

[6] I. Sahul Hamid, and S. Ashok Kumar, "Equitable irregular edge-weighting of graphs", SUT Journal of Mathematics, 46, No. 1 (2010), 79 - 91.

[7] S. Saravanakumar, "Coprime Irregular graphs", IJEAT, 8, Vol.9, Issue-4S4, December 2019. 